\title{
Emotional Reactions to Sounds without Meaning
}

\author{
Daniel Västfjäll \\ Department of Behavioural Science and Learning, Linköping University, Linköping, Sweden \\ Email: daniel.vastfjall@liu.se \\ Received May $19^{\text {th }}, 2012$; revised June $18^{\text {th }}, 2012$; accepted July $16^{\text {th }}, 2012$
}

\begin{abstract}
The present research examined the relationship between emotional reactions to sounds without meaning (tone and noise complexes) and objective sound descriptors. Two experiments showed that the core affect dimensions valence and activation were related to perceived loudness (intensity) and sharpness (perceived high frequency content), respectively. These results can be used as design criteria for emotion induction with sounds, implementation of emotional sounds in products, as well as in research on environmental noise perception.
\end{abstract}

Keywords: Emotion; Sound

\section{Introduction}

Early research by Wundt suggested that emotional reactions to auditory events could be mapped onto a pleasantness-unpleasantness (Lust-Unlust) dimension (Wundt, 1924). Later studies on emotional reactions to non-musical, non-vocal sounds have almost exclusively studied positive-negative responses (Todd, 2001; Vitz, 1973), even though Wundt himself concluded that emotional reactions to auditory rhythms needed to be described by additional dimensions (strain-relaxation and excitement-calmness). More recent studies on the relationship between emotional responses and sound characteristics have focused on a single affective state annoyance. Annoyance has been shown to correlate moderately with descriptors of physical characteristics such as equivalent $\mathrm{dB}(\mathrm{A})$ level for community noise, and with other psychoacoustic dimensions such as perceived sharpness and roughness for specific sound sources (Berglund \& Lindvall, 1995; Berglund, Hassmén, \& Preis, 2002; Zwicker \& Fastl, 1999).

Other research have demonstrated that two dimensions, valence and activation, are suited to describe emotional reactions to sounds (Björk, 1985). Bradley and Lang (2000) found that self reported emotional reactions to 60 natural sounds were scattered in a two-dimensional space of valence (pleasantnessunpleasantness) and activation (arousal) (but see Stevenson \& Jameson, 2008 for a discrete emotional account of these sounds). Moreover, the reactions were clustered along two axes, one stretching from low activation and neutral valence to unpleasant high activation (avoidance), and the other one from low activation and neutral valence to pleasant high activation (approach). Importantly, Bradley and Lang found that valence ratings was very weakly related to sound level $(\mathrm{r}=0.07)$ and activation ratings was moderately related $(r=0.38)$. However, these correlations only accounted for $14 \%$ of the variance. Affective reactions to these sounds must therefore be related to

\footnotetext{
${ }^{1}$ Affective meaning is defined as an evaluation of auditory events where the object/activity creating the sound is easily identified (Ballas, 1993), and that this activity/object is perceived as positive-negative. Furthermore it is the object/activity, rather that the acoustic information, that creates the affective reaction
}

other physical sound descriptors than sound level and/or other psychological characteristics not captured by physical descriptors (Asutay \& Västfjäll, 2012; Asutay et al., 2012). Bradley and Lang used recordings of a number of everyday sounds sources such as recordings of a dog barking, cries from an amusement park etc., why they could conclude that the observed reactions were due to other aspects of the stimuli (i.e. affective meaning ${ }^{1}$ ).

The present research complements the research by Bradley and Lang by focusing on physical sound determinants of emotional reactions. Results from such research are important for many applications including emotion induction with sounds, assessment of subjective noise reactions, prediction of subjective noise experience, sound design, auditory interfaces, and development of new sound abatement approaches (Desmet, 2002; Picard, 1997; Västfjäll et al., 2002). A slightly different approach than that of Bradley and Lang's is therefore used in the present research. Rather than using everyday sounds with easily identified meaning (such as recordings of people, animals, activities), the present research focused sounds devoid of emotional meaning (noise and tone complexes) induced through activation of episodic memories or similar mechanisms (Juslin \& Västfjäll, 2008). Even though everyday emotional reactions are related to both sound characteristics and the appraisal of the sound/sound source (Asutay \& Västfjäll, 2012; Tajadura, Väljamäe, Asutay, \& Västfjäll, 2010, Tajadura, Larsson, Väljamäe, Västfjäll, \& Kleiner, 2010), it may be desirable to first establish a relationship between sound characteristics and emotional reactions.

The aim of the present research is therefore to study 1) if emotional reactions to tone and noise complexes that vary in the two-dimensional emotional experiential space; 2) to find self-reported and physical correlates to valence and activation. A related aim is to show that auditory dimensions other than loudness or sound level influence emotional reactions to sounds (Zwicker \& Fastl, 1999).

In Experiment 1, participants either rated their emotional reactions to 16 tone and noise complexes or rated the perceptual or psychoacoustic qualities of the same sounds. On basis of the results of Experiment 1, Experiment 2 used experimentally 
manipulated sounds to further investigate physical determinants of valence and activation.

\section{Experiment 1}

The first experiment aimed at investigating the relationship between emotional reactions and sound characteristics. An exploratory approach was taken where 40 participants rated their emotional reactions to stationary sounds. Twenty additional participants rated the sounds in terms of their perceptual properties.

Participants. Sixty undergraduates at Chalmers University of Technology, Göteborg, Sweden, an equal number of men and women, participated on a voluntary basis. They were compensated with the equivalence of US\$10. Their mean age was 26.1 years (SD 4.1). All participants had normal hearing as determined by an audiogram.

Measures. The affect measures consisted of two bipolar scales each defined by three adjective pairs found in previous research to tap valence and activation, respectively (Västfjäll et al., 2002; Västfjäll \& Gärling, 2007). Sleepy-awake, dull-peppy, and passive-active were used to define the activation scale, displeased-pleased, sad-glad, and depressed-happy were used to define the valence scale. Numbers ranging from -4 over 0 to 4 were typed beneath the three adjective pairs defining a scale. Participants were requested to circle the number that corresponded to their feeling.

From previous research on auditory event evaluation (Björk, 1985; von Bismark, 1974; Solomon, 1958), a set of sensory adjectives were identified and selected. These included (translated to English) hard, strong, low, loud, clear, tonal, even, high in frequency, low in frequency, rough, soft, regular, irregular, weak in tone, harsh, natural, artificial, tiring, sharp, edgy, blunt, strong in tone, presence of extraneous sounds, and balance between left and right ear.

Stimuli and presentation. Sixteen binaural sounds varying in psychoacoustical qualities were used. The sounds were tone and noise complexes and a pilot rating experiment suggested that they were not systematically identified as a having a specific meaning. Psychoacoustic metrics were calculated for all sounds using a HEAD Acoustics Artemis analysis system on a PC. The sounds varied in psychoacoustical properties such as loudness (intensity), roughness (frequency or amplitude modulations between $20-70 \mathrm{~Hz}$ ), sharpness (high frequency components), fluctuation strength (amplitude and frequency modulations below $20 \mathrm{~Hz}$ ), and tonal content (tone-to-noise ratio; see Zwicker \& Fastl, 1999 for an overview of these metrics). The sounds were presented in an acoustically well-damped room over Alpine loudspeakers, using cross cancellation technique to maintain binaural information.

\section{Procedure}

Emotion ratings. Participants $(\mathrm{n}=40)$ arrived individually to the laboratory. After having been seated participants listened to the first of in all 16 sounds sequentially presented. Each sound was presented for 2 minutes. Participants were asked to rate their affective reactions on the adjective scales by checking each scale to indicate the degree to which the adjective described how they felt at the time of listening to the sound.

Following the procedure devised by Bradley and Lang (2000), participants were instructed to refer how they felt when listening to the sound. The moment participants were asked to refer to when rating their affective reactions was indicated by a "rating probe" consisting of a blinking arrow on a computer screen. The rating probe was displayed approximately in the middle of the duration of each sound.

After listening to a sound, participants were instructed relax and try to return to a neutral affective state. When participants felt relaxed, they were instructed to rate how they felt on the two adjective scales. After this they continued with the next sound by clicking a button on the computer.

Sensory ratings. 20 separate participants rated the sensory characteristics of the sounds. The procedure was similar to the affective ratings condition. Sounds were presented in different random orders for each participant. One minute was allowed between each sound. Participants could indicate any number from 0 (not at all) to 8 (very much) for each of the adjectives. The procedure took in total approximately one hour.

For each participant a different random order of the sounds was generated. The participants needed about sixty minutes to complete the ratings. After listening to the sixteen sounds, participants were debriefed, compensated and thanked for their participation.

\section{Results and Discussion}

First, emotional reactions were examined. Valence and activation were relatively independent $(r=0.09, p>0.05)$. Figure 1 shows that the sounds induced variations in emotional reactions in terms of activation and valence. This was substantiated by a within-subject ANOVA on activation, $F(2.12$, $49.66)=12.44, p<0.001$, and on valence, $F(2.77,46.82)=$ $20.10, p<0.001$.

To assess the relationship between the psychoacoustic metrics and emotional reactions multiple regression analyses with either the mean valence or mean activation index as dependent variable was performed. The analysis for the activation index showed that sharpness contributed significantly $(\beta=0.68, p<$ 0.01 ) for an $R_{a d j}^{2}$ of $0.42, F(2,14)=11.98, p<0.01$.

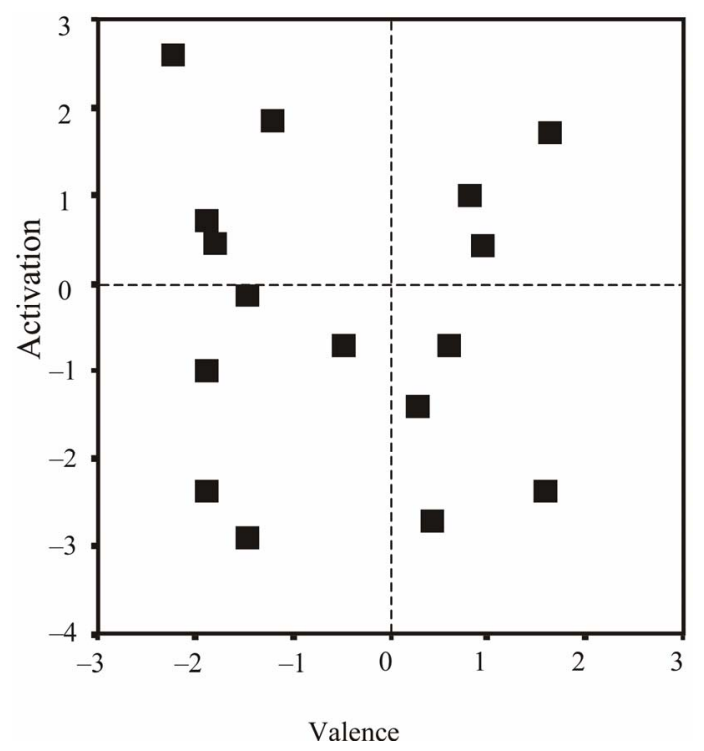

Figure 1.

Emotional reactions to 16 tone and noise complexes along the valence and activation dimensions (Experiment 1). 
For the regression analysis of the valence index the loudness $(\beta=-0.89, p<0.001)$ and roughness $(\beta=0.38, p<0.05)$ metrics contributed significantly giving an $R_{\text {adj }}^{2}$ of $0.81, F(3,13)=$ $33.42, p<0.01$.

To further corroborate these results, sensory ratings from the separate sample were investigated. A PCA was first performed on the correlations between the mean ratings over individuals for each sound to determine the underlying perceptual dimensions. The PCA resulted in five factors with eigenvalues larger than 1.0 , that together accounted for $76 \%$ of the variance. Adjectives denoting loudness loaded on the first factor, adjectives describing mainly sensory sharpness loaded on the second factor, adjectives describing fluctuation and modulation loaded on the third factor, adjectives describing tonal content loaded on the fourth factor, and finally adjectives concerning the naturalness vs. artificiality of the sounds loaded on the fifth factor. From the PCA five indices of loudness, sharpness, fluctuation strength, tonal content, and naturalness were formed by summing with the appropriate sign.

Next, multiple regression analyses were performed with each of the affect indices as dependent variables. Sharpness $(\beta=$ $0.47, p<0.05)$ and tonal content $(\beta=-0.43, p<0.05)$ indices were reliably related to the activation index, $R_{\text {adj }}^{2}=0.53, \mathrm{~F}(3$, $13)=12.85, p<0.01$, indicating that activation increases with increasing sharpness and decreases with increasing tonal content. Loudness $(\beta=-0.51, p<0.01)$ and naturalness $(\beta=0.32$, $p<0.05)$ were reliably related to valence, $R_{a d j}^{2}=0.66, \mathrm{~F}(3,13)$ $=18.83, p<0.01$.

The results of the first experiment showed that tone and noise complexes varied in both valence and activation. More importantly, the results suggested that valence and activation reactions differed in their determinants. Activation was related to rated or perceived tonal content and sharpness, whereas valence was associated with perceived loudness, roughness, and naturalness.

To further study these relationships, Experiment 2 employed experimentally manipulated sounds.

\section{Experiment 2}

\section{Method}

Participants. 16 undergraduates, 7 female and 9 males, voluntarily participated in the experiment. Their mean age was 22.3 (SD, 2.12). All reported having normal hearing.

Measures. The affect rating scales from Experiment 1 was used.

\section{Stimuli and Presentation}

Activation manipulation. Since the first experiment showed that activation was related to perceived sharpness of the sound a set of five sounds varying from strong low-frequency tonal content strong to high frequency content was created using signal processing. A tone-noise complex was used as the base stimulus (reference sound). From this sound a number of modified versions were created where the fundamental frequency and/or harmonics or the noise spectrum were changed. The modifications for strong tonal low frequency content were an

\footnotetext{
${ }^{2} \mathrm{~A}$ pilot experiment indicated that the perceived sharpness/tonal content varied as predicted, whereas perceived loudness remained constant.
}

amplification of the fundamental frequency $(100 \mathrm{~Hz})$ of 6 and $12 \mathrm{~dB}$, respectively. To increase the high frequency content of the sound, a high-pass filter was used to amplify noise and tones above $3000 \mathrm{~Hz}$ with 6 and $12 \mathrm{db}$, respectively. Finally, all sounds were equalized to the same loudness level and were replayed to participants at $60 \mathrm{dBA}^{2}$.

Valence manipulation. For the valence manipulation only loudness was changed. The same reference sound that was used for the activation manipulation was again used, but replayed at five different sound levels $(40,50,60,70$, and $80 \mathrm{dBA})$.

All stimuli were generated using digital signal processing software and were digitally stored on a computer. Stimulus presentation was made on computers using an experiment program. The sounds were delivered via Stax electrostatic headphones.

Procedure. Participants performed the experiment individually or in groups of maximum three persons at a time. Upon arrival to the laboratory, participants were first instructed how to use the scales and equipment. They were instructed that they would perform ratings of their reactions to various sounds. Participants then listened to and rated five trial sounds (other than the test stimuli). After that they rated the five trial sounds, participants continued with remaining two sound blocks. The blocks and order within blocks were counterbalanced across participants. When participants had rated all the sounds, they were debriefed, compensated, and thanked for their participation.

\section{Results and Discussion}

Activation manipulation. The within-subjects ANOVAs for activation, $\mathrm{F}(2.31,34.66)=46.01, p<0.001$, was as expected significant. As may be seen in Figure 2, activation increases with increasing sharpness. The ANOVA for valence was not significant $\mathrm{F}(1.44,21.66)=1.09, p>0.05$.

Valence manipulation. The within-subjects ANOVA for valence was significant, $\mathrm{F}(2.86,42.96)=24.12, p<0.001$ (Figure 3). The ANOVA for activation was also significant, $\mathrm{F}(1.99,37.97)=4.01, p<0.05$. A contrast however showed that only the $80 \mathrm{dBA}$ vs $40 \mathrm{dBA}$ was significantly different.

In line with experiment 1 and previous findings (Västfjäll et al., 2002), Experiment 2 yielded support for the idea that valence reactions are mainly affected by loudness and the activation dimension by perceived sharpness of the sound.

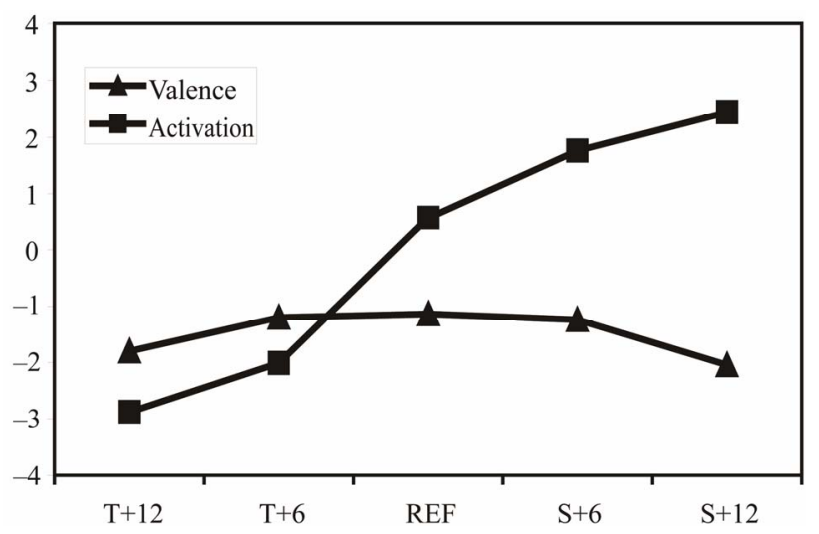

Figure 2.

Valence and activation ratings for sharpness modifications (Experiment 2). 


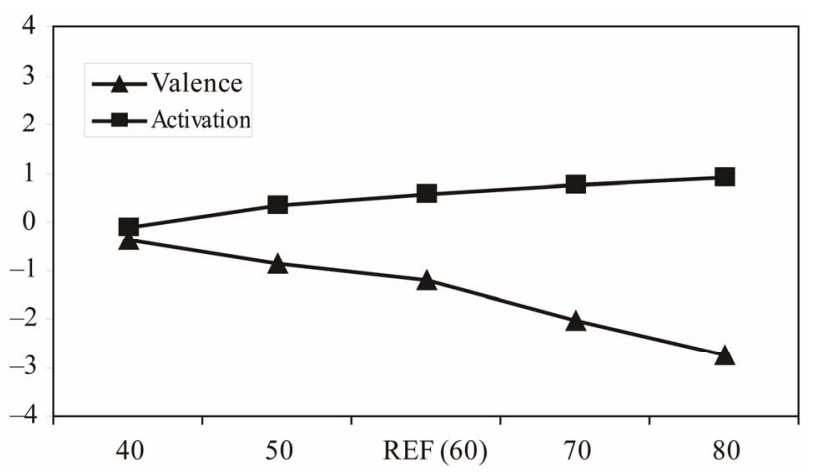

Figure 3.

Valence and activation ratings for loudness changes (Experiment 2).

\section{General Discussion}

The present research showed that the valence and activation dimensions of auditory-induced emotions are related to different physical characteristics. Valence is primarily determined by the perceived loudness and activation by the perceived sharpness of the sound. These findings are line with other research showing that tonal sounds decrease wakefulness and that sound level is related to annoyance (see Berglund \& Lindvall, 1995 for an overview). The present research however goes beyond most research on noise annoyance in showing that it is useful to decompose emotional reactions to sounds into the two core affect dimensions valence and activation (see also Asutay et al., 2012).

The sounds used in the present research were all static, stationary sounds devoid of meaning that may modulate emotional experience. Thus, the present research complement previous research on emotional reactions to everyday natural sounds (Bradley \& Lang, 2000) by showing that physical characteristics may be a good predictor of emotional reactions to sounds when they carry little affective meaning, but still induce affect in the listener. It is also possible that physical characteristics uncovered here may be good predictors of emotional reactions to sounds that initially carry emotional meaning, but that through habituation is reduced (like traffic noise). Other research also suggests that objective measures may predict emotional reactions to any set of sounds that do not vary drastically in meaning (Västfjäll et al., 2002; Asutay et al., 2012). It should however be noted that for sounds that do vary in emotional meaning, physical characteristics will likely be much less predictive of the emotional reaction. In music, for instance, musical structure is very important to convey emotions, but much less important for inducing emotion (Juslin \& Västfjäll, 2008).

The present results can be used as design criteria for emotion induction studies using sound (Västfjäll, 2002), design of emotive sounds for various applications (Norman, 2002; Tajadura \& Västfjäll, 2008), affective computing (Picard, 1997), as well as a base for future studies on emotions in noise perception (Västfjäll et al., 2002). An important task for future research is to investigate if the relationship between experienced affect and objective sound characterization holds for a wider range of sounds and situations (e.g. natural soundscapes).

\section{Acknowledgements}

This research was financially supported by Swedish Council for Working Life and Social Research.

\section{REFERENCES}

Asutay, E., \& Västfjäll, D. (2012). Perception of loudness is influenced by emotion. PLOS ONE, 7, e38660.

doi:10.1371/journal.pone. 0038660

Asutay, E., Västfjäll, D., Tajadura-Jimenez, A., Genell, A., Bergman, P., \& Kleiner, M. (2012). Emoacoustics: A study of the psychoacoustical and psychological dimensions of emotional sound design. Journal of the Audio Engineering Society, 60, 21-28.

Ballas, J. A. (1993). Common factors in the identification of an assortment of brief everyday sounds. Journal of Experimental Psychology: Human Perception and Performance, 19, 250-267. doi:10.1037/0096-1523.19.2.250

Berglund, B., Hassmén, P., \& Preis, A. (2002). Annoyance and spectral contrast are cues for similarity and preference of sounds. Journal of Sound and Vibration, 250, 53-64. doi:10.1006/jsvi.2001.3889

Berglund, B., \& Lindvall, T. (1995). Community noise. Archives of the Center for Sensory Research, 2. Stockholm: Stockholm University.

Björk, E. A. (1985). The perceived quality of natural sounds. Acustica, 57, 185-188.

Bradley, M. M., \& Lang, P. J. (2000). Affective reactions to acoustic stimuli. Psychophysiology, 37, 204-215. doi:10.1111/1469-8986.3720204

Desmet, P. (2002). Designing emotions. Delft: Delft University of Technology.

Juslin, P. N., \& Västfjäll, D. (2008). Emotional responses to music: The need to consider underlying mechanisms. Behavioral Brain Science, 31, 559-621.

Norman, D. A. (2002). Emotion and design: Attractive things work better. Interactions Magazine, 9, 36-42.

Picard, R. (1997). Affective computing. Cambridge: MIT Press.

Solomon, L. N. (1958). Semantic approach to the perception of complex sounds. Journal of the Acoustical Society of America, 30, 421425. doi:10.1121/1.1909632

Stevenson, R. A., \& James, T. W. (2008). Affective auditory stimuli: Characterization of the International Affective Digitized Sounds (IADS) by discrete emotional categories. Behavior Research Methods, 40, 315-321. doi:10.3758/BRM.40.1.315

Tajadura, A., \& Västfjäll, D. (2008). Auditory induced-emotion: A neglected channel for communication in HCI. In Affect and Emotion in HCI. In C. Peter, \& B. Russell (Eds.), Berlin: Springer Verlag. doi:10.1007/978-3-540-85099-1 6

Tajadura-Jimenez, A., Väljamäe, A., Asutay, E., \& Västfjäll, D. (2010). Embodied auditory perception: The emotional Impact of approaching and receding sound sources. Emotion, 10, 216-229. doi: $10.1037 / \mathrm{a} 0018422$

Tajadura-Jimenez, A., Larsson, P., Väljamäe, A., Västfjäll, D., \& Kleiner, M. (2010). The influence of auditory space on emotional response to sounds. Emotion.

Todd, N. (2001). Evidence for a behavioral significance of saccular acoustic sensitivity in humans. Journal of the Acoustical Society of America, 110, 380-390. doi:10.1121/1.1373662

Västfjäll, D. (2002). Emotion induction through music: A review of the musical mood induction procedure. Musicae Scientiae, 6, 171-203.

Västfjäll, D., \& Gärling, T. (2007). Validation of short self-report measure of core affect. Scandinavian Journal of Psychology, 48, 233-238. doi:10.1111/j.1467-9450.2007.00595.x

Västfjäll, D., Gulbol, M.-A., Kleiner, M., \& Gärling, T. (2002). Affective reactions to- and evaluations of interior and exterior vehicle auditory quality. Journal of Sound and Vibration, 255, 501-518. doi:10.1006/jsvi.2001.4166

Västfjäll, D., Friman, M., Gärling, T., \& Kleiner, M. (2002). The measurement of core affect: A Swedish self-report measure. Scandinavian Journal of Psychology, 43, 19-32. doi:10.1111/1467-9450.00265

Vitz, P. C. (1973). Preference for tones as a function of frequency and intensity. Perception and Psychophysics, 11, 84-88. doi:10.3758/BF03212689

Wundt, W. (1924). An introduction to psychology. London: Allen \& Unwin.

Zwicker, E., \& Fastl, H. (1999). Psychoacoustics—Facts and models. (2nd ed.). Heidelberg: Springer-Verlag. 